\title{
3-Hydroxykynurenine Toxicity on the Rat Striatum In Vivo
}

\author{
Yasuhiro Nakagami, Hiroshi Saito and Hiroshi Katsuki* \\ Department of Chemical Pharmacology, Faculty of Pharmaceutical Sciences, The University of Tokyo, 7-3-1 Hongo, Bunkyo-ku, Tokyo 113, Japan \\ Received March 15, 1996 Accepted April 18, 1996
}

\begin{abstract}
Hydroxykynurenine (3-HK) is a tryptophan metabolite whose level in the brain is elevated under several pathological states including Huntington's disease. In the present study, we examined the possible toxicity of 3-HK by injection of this substance into rat brain. Intrastriatal injection of 3-HK ( 50 $\mathrm{nmol}$ ) induced tissue damage around the injected site. Quinolinic acid (QA) at $200 \mathrm{nmol}$ also induced tissue damage, which was comparable in size to that induced by $50 \mathrm{nmol} \mathrm{3-HK}$. Co-administration of MK-801 significantly reduced QA-induced lesion, but failed to prevent 3-HK-induced lesion. On the other hand, the antioxidant $\mathrm{N}$-acetyl-L-cysteine $(10 \mathrm{nmol})$ reduced 3-HK-induced damage. Thus, 3-HK may be involved in brain pathogenesis by providing oxygen radicals.
\end{abstract}

Keywords: 3-Hydroxykynurenine, Quinolinic acid, Huntington's disease

It is considered that a metabolic alteration in the synthesis or degradation of endogenous neurotoxic substances may explain the pathological mechanisms of several neurodegenerative disorders. The kynurenine pathway is the major metabolic pathway of the amino acid tryptophan. This pathway is important in the formation of nicotinic acid and nicotinamide, but also has been implicated in brain pathogenesis, since it generates possible neurotoxic compounds as metabolic intermediates. 3-Hydroxykynurenine (3-HK) is one of the metabolites of the kynurenine pathway. 3-HK shows potent toxicity on a neuronal hybrid cell line $(1,2)$. Moreover, it has been reported that the levels of 3-HK are elevated in the brains of patients with Huntington's Disease (HD) (3-5), hepatic encephalopathy (6) and AIDS (7). Therefore, 3-HK might be involved in the pathogenesis of several diseases. However, to date, there is no literature demonstrating toxic actions of 3-HK on brain tissues and cells in vivo. On the other hand, quinolinic acid (QA), another intermediate of the kynurenine pathway, has been well investigated. Especially, QA acts as an excitotoxin for specific neuronal groups and can produce striatal lesions similar to those found in HD brain $(8,9)$, but there is no convincing evidence that $\mathrm{QA}$ concentration is increased in HD brain (10). The aim of the present study is to reveal whether 3-HK can induce potent pathological changes compared to QA in vivo.

\footnotetext{
* To whom correspondence should be addressed.
}

Male Wistar rats (Nippon Bio-supp. Center, Tokyo) weighing 190-210 g were anesthetized with ketamine (41 $\mathrm{mg} / \mathrm{kg})$ and xylazine $(0.35 \mathrm{mg} / \mathrm{kg})$ and then placed in a stereotaxic frame with a nose bar. The skin was removed to expose the skull and a $0.9-\mathrm{mm}$ diameter hole was made using a drill to permit drug injection. Drugs were injected at a rate of $0.2 \mu \mathrm{l} / \mathrm{min}$ in the left and/or right striatum (coordinates: AP $+0.7 \mathrm{~mm}$ from the bregma, LM 2.8 $\mathrm{mm}$ from the midline, DV $4.6 \mathrm{~mm}$ ) in a volume of $1 \mu \mathrm{l}$, using a microsyringe fixed on the stereotaxic frame. The needle was left for $1 \mathrm{~min}$ after the injection, then slowly withdrawn, and the skin was sutured. After surgery, animals were maintained with food and water ad libitum. One week after injection, the animals were again anesthetized and first perfused through the heart with phosphate-buffered saline (PBS) and then subsequently with $4 \%$ paraformaldehyde solution in $0.1 \mathrm{M}$ phosphate buffer (pH 7.4). Brains were postfixed overnight at $4^{\circ} \mathrm{C}$ in paraformaldehyde/phosphate buffer and then immersed in 20\% sucrose/phosphate buffer ( $\mathrm{pH} 7.4$ ).

For histological evaluations, brains were frozen at $-20^{\circ} \mathrm{C}$ and $10-\mu \mathrm{m}$ coronal sections were cut every $100 \mu \mathrm{m}$ using a Reichert Jung Cryostat (Heidelberg, Germany) and thaw-mounted onto silane-coated slides. Nissl staining was performed with $0.5 \%$ cresyl violet solution after rinsing the sections twice for 2 min with PBS.

3-HK, QA and $N$-acetyl-L-cysteine were purchased from Sigma Chemical Co. (St. Louise, MO, USA), and $(+)-M K-801$ hydrogen maleate was from Research 
Biochemicals, Inc. (Natick, MA, USA). All materials for intrastriatal injections were dissolved in dimethyl sulfoxide (DMSO), because 3-HK was not readily soluble. The maximal soluble concentration of $3-\mathrm{HK}$ was $50 \mathrm{mM}$, and therefore the highest dose of 3-HK tested in the present study was $50 \mathrm{nmol}$. Control rats received an injection of the same volume of DMSO alone.

We determined the effects of all drugs 7 days after intrastriatal injection, since other studies demonstrating striatal lesions by several kinds of neurotoxins have also employed this time period $(11,12)$. Intrastriatal injection of 3-HK induced lesion around the injected area. As shown in Fig. 1A, lesion induced by $50 \mathrm{nmol} 3-\mathrm{HK}$ was clearly identified by the cavity. Surrounding this cavity,

A
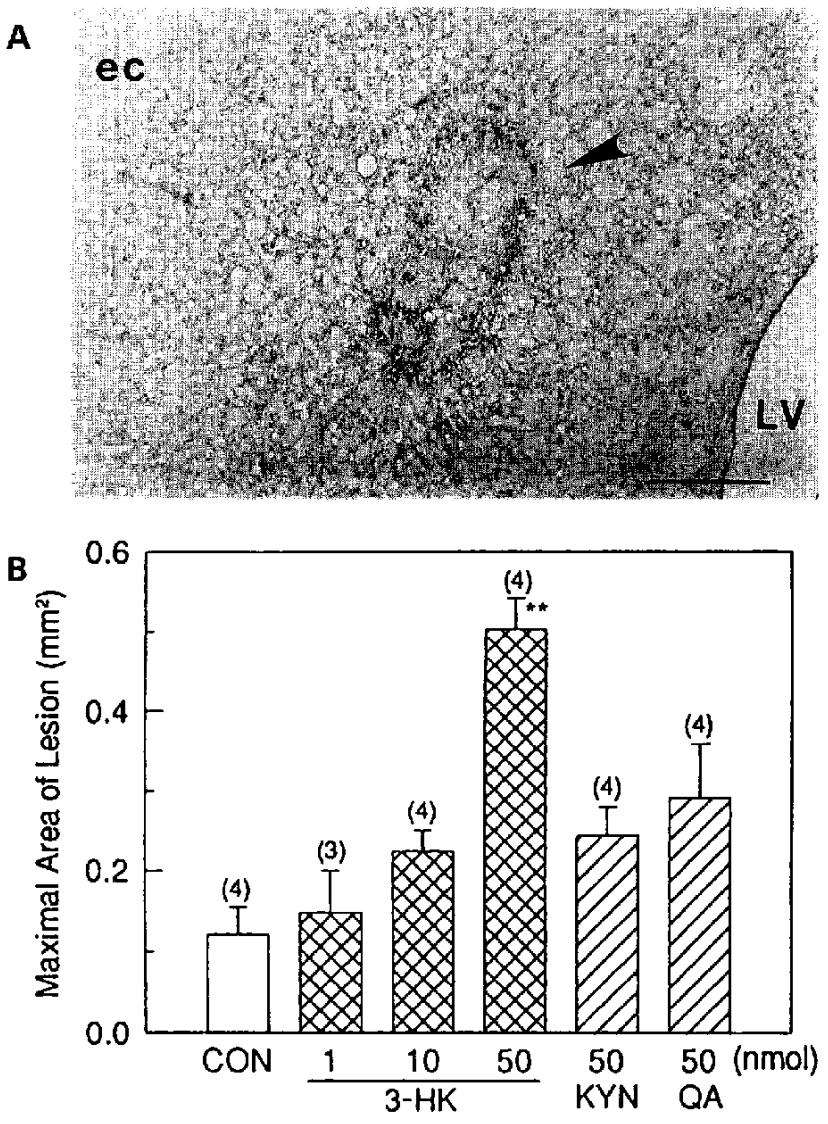

Fig. 1. The cytotoxicity of 3-hydroxykynurenine (3-HK). A: A Nissl-stained coronal section of the right striatum (AP $+0.7 \mathrm{~mm}$ from the bregma) 7 days after injection of $50 \mathrm{nmol} 3-\mathrm{HK}$. Injection of 3-HK caused a lesion (denoted by an arrowhead) characterized by the presence of a cavity, surrounded by necrotic and reactive gliosis. Abbreviations: LV, lateral ventricle; ec, external capsule. Bar $=500$ $\mu \mathrm{m}$. B: Dose-dependent increase in the size of lesion 7 days after intrastriatal injection of $3-\mathrm{HK}$ and comparison with kynurenine (KYN) and quinolinic acid (QA). Control rats were given only vehicle ( $1 \mu$ l DMSO). Results are expressed as a mean \pm S.E.M. with the number of subjects in parentheses. ${ }^{* *} \mathrm{P}<0.01$ vs vehicle control (CON), by Dunnett's multiple range test. there was an area of necrotic and reactive gliosis where darkly stained cells were densely packed. For quantitative evaluation, Nissl-stained sections were photographed by 40-fold magnifications, and lesion areas surrounded by glial lineage were quantified using a digitizer. We estimated the extent of the lesions by the maximal lesion area in successive sections. When $1 \mu \mathrm{l}$ of PBS was injected, no cavity was present around the injected area, which indicated that damage by the injection procedures was minimal under our experimental conditions. Injection of $1 \mu 1 \mathrm{DMSO}$, used as a vehicle, did induce slight tissue damage (Fig. 1B, white column), which was inevitable, probably due to the properties of DMSO as a solvent. On the other hand, 3-HK dissolved in the same volume of DMSO, markedly increased the area of lesion in a dosedependent manner (Fig. 1B). Two other tryptophan metabolites were also tested and compared with the toxicity of 3-HK at the same dose of $50 \mathrm{nmol}$. Kynurenine, an immediate precursor of 3-HK in the kynurenine pathway, showed no toxic effect as compared to the vehicle control. The excitotoxin QA also showed no significant toxicity at $50 \mathrm{nmol}$ : at a higher dose of $200 \mathrm{nmol}$, QA induced tissue damage comparable in size to that induced by $50 \mathrm{nmol} \mathrm{3-HK}$ (Fig. 2). These results indicate that 3 HK is a potent toxic substance on brain tissue. However, no animals died after surgery, and we did not observe any abnormal behaviors after 3-HK injection under the present experimental conditions.

QA is known to exert its toxic actions through the activation of the $N$-methyl-D-aspartate (NMDA) subtype of glutamate receptors (8). To clarify whether the same mechanisms are involved in the toxicity of $3-\mathrm{HK}$, the

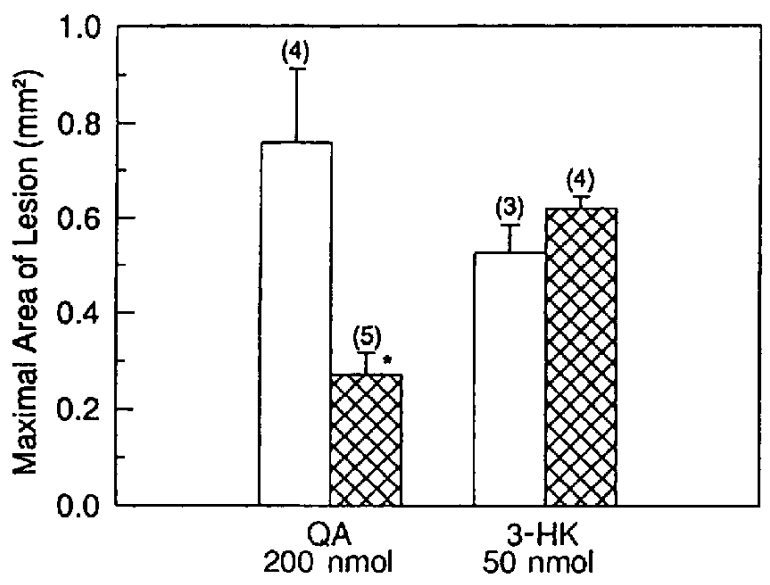

Fig. 2. The effect of MK-801 on quinolinic acid (QA)- and 3hydroxykynurenine (3-HK)-induced lesions 7 days after intrastriatal injection. MK-801 was simultaneously administered with QA (200 nmol) or 3-HK $(50 \mathrm{nmol})$. MK- $801,10 \mathrm{nmol}$. Results are expressed as a mean \pm S.E.M. with the number of subjects in parenthses. ${ }^{*} \mathrm{P}<0.05$ vs no MK-801 ( $\left.\mid\right)$, by Student's $t$-test. 
non-competitive NMDA-receptor antagonist MK-801 was used. As presented in Fig. 2, co-administration of 10 nmol MK-801 significantly reduced QA-induced damage in the striatal tissue. However, the same dose of MK-801 did not prevent the tissue damage induced by $3-\mathrm{HK}$. Rather, MK-801 seemed to slightly exacerbate the toxic effect of 3-HK, but the difference did not reach statistical significance. In previous studies using a cell line, it has been demonstrated that the generation of hydrogen peroxide may mediate the cytotoxicity of 3 -HK $(1,2)$. Therefore, we tested the effect of the antioxidant $N$ acetyl-L-cysteine (13) against 3 -HK toxicity. We found that simultaneous application of $10 \mathrm{nmol} \mathrm{N}$-acetyl-L-cysteine largely and significantly attenuated the tissue damage induced by $50 \mathrm{nmol} 3-\mathrm{HK}$ (Fig. 3).

We have demonstrated in the present study, to our knowledge, for the first time that $3-\mathrm{HK}$ is a potent toxic substance on brain tissue. QA is a widely studied tryptophan metabolite as an excitotoxin, which induces pathological changes in the striatum resembling some of the characteristics of HD brain $(8,9)$. Here $3-\mathrm{HK}$ was found to be more potent than QA in inducing striatal lesion, when compared at the same dose. Moreover, the mechanisms of toxicity are quite different between these substances. QA is known to exert its toxic actions by excitotoxic mechanisms, that is, by activating the NMDA subtype of glutamate receptors and allowing abnormal $\mathrm{Ca}^{2+}$ influx into neuronal cells (8). Consistent with this view, we observed that co-administration of MK-801 completely blocked QA toxicity on striatal tissue. However, 3-HK toxicity was not affected by MK-801

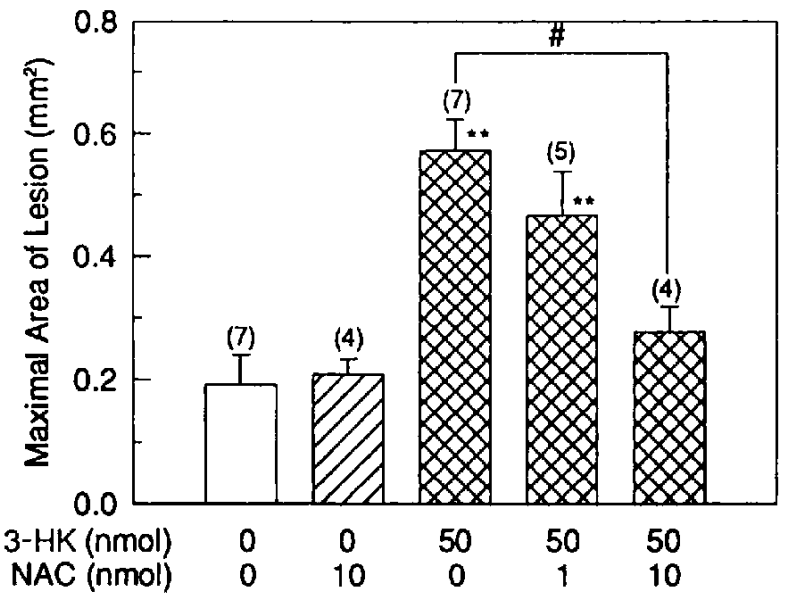

Fig. 3. Protective effect of $\mathrm{N}$-acetyl-L-cysteine (NAC) against 3hydroxykynurenine (3-HK)-induced lesion 7 days after intrastriatal injection. NAC at the indicated doses was simultaneously administered with 3-HK. Results are expressed as a mean \pm S.E.M. with the number of subjects in parentheses. ${ }^{* *} \mathrm{P}<0.01$ vs vehicle control, ${ }^{\sharp} \mathrm{P}<0.05$ vs 3 -HK alone; by Dunnett's multiple range test. treatment.

On the other hand, we found that the toxicity of 3-HK could be prevented by the antioxidant $N$-acetyl-L-cysteine. Our results suggest that oxygen radicals are involved in the 3-HK toxicity, although we cannot determine the species of oxygen radicals responsible for the observed toxicity. In this context, the studies of Eastman and Guilarte $(1,2)$ are notable, where the toxicity of $3-\mathrm{HK}$ has been demonstrated on the neuronal hybrid cell line N18-RE105 . They showed that the toxicity of $3-\mathrm{HK}$ was attenuated by catalase or glutathione and suggested that hydrogen peroxide, generated as a result of the autooxidation of $3-\mathrm{HK}$, is responsible for the cytotoxicity.

The present study raises the possibility that $3-\mathrm{HK}$ is at least in part involved in the several pathological conditions that are associated with various neurological dysfunctions. For example, the levels of this endogenous compound is reported to be elevated in HD brain (3-5). It has been also reported that vitamin $\mathrm{B}_{6}$ deficiency during the perinatal period results in gross neurological symptoms (14) and increased levels of 3-HK in rat brain (15). Hepatic encephalopathy is associated with increased concentrations of brain 3-HK (6). Increased levels of 3-HK in the brains of AIDS patients have been recently reported, where the increase was more prominent in the brains of patients with dementia (7). 3-HK might exert its pathogenic actions in these various situations.

We did not observe any abnormal behaviors in rats injected with 3-HK, such as tremor, catalepsy, and nocturnal locomotor activity. This may be due to the fact that 3-HK was poorly soluble in conventional vehicles for microinjection; therefore, that restricted the dose of 3$\mathrm{HK}$, and the resultant small volumes of lesions were not enough to induce behavioral syndromes. Further studies that employ different approaches such as chronic treatment of low dose 3-HK are certainly required. Nevertheless, the present single-injection study revealed clearly that this endogenous substance could be a potent risk factor in pathological conditions in the brain.

\section{REFERENCES}

1 Eastman CL and Guilarte TR: Cytotoxicity of 3-hydroxykynurenine in a neuronal hybrid cell line. Brain Res 495, 225-231 (1989)

2 Eastman CL and Guilarte TR: The role of hydrogen peroxide in the in vitro cytotoxicity of 3-hydroxykynurenine. Neurochem Res 15, $1101-1107$ (1990)

3 Pearson SJ and Reynolds GP: Increased brain concentration of a neurotoxin, 3-hydroxykynurenine, in Huntington's disease. Neurosci Lett 144, 199-201 (1992)

4 Reynolds GP and Pearson SJ: Increased brain 3-hydroxykynurenine in Huntington's disease. Lancet 2, 979-980 (1989)

5 Guilarte TR and Eastman CL: Is 3-hydroxykynurenine an endogenous neurotoxin in Huntington's disease? J Neurol Sci 
116, 227-228 (1993)

6 Pearson SJ and Reynolds GP: Determination of 3-hydroxykynurenine in human brain and plasma by high-performance liquid chromatography with electrochemical detection. Increased concentration in hepatic encephalopathy. J Chromatogr 565, 436-440 (1991)

7 Sardar AM, Bell JE and Reynolds GP: Increased concentration of the neurotoxin 3-hydroxykynurenine in the frontal cortex of HIV-1-positive patients. J Neurochem 64, 932-935 (1995)

8 DiFiglia M: Excitotoxic injury of the neostriatum: a model for Huntington's disease. Trends Neurosci 13, 286-289 (1990)

9 Beal MF, Kowall NW, Ellison DW, Mazurek MF, Swartz KJ and Martin JB: Replication of the neurochemical characteristics of Huntington's disease by quinolinic acid. Nature 321, $168-171$ (1986)

10 Reynolds GP, Pearson SJ, Halket J and Sandler M; Brain quinolinic acid in Huntington's disease. J Neurochem 50, $1959-1960(1988)$
11 Brouillet E, Jenkins BJ, Hyman BT, Ferrante RJ, Kowall NW, Srivastava R, Roy DS, Rosen BR and Beal MF: Age-dependent vulnerability of the striatum to the mitochondrial toxin 3nitropropionic acid. J Neurochem 60, 356-359 (1993)

12 Beal MF, Kowall NW, Swartz KJ, Ferrante RJ and Martin JB: Systemic approaches to modifying quinolinic acid striatal lesions in rats. J Neurosci 8, 3901-3908 (1988)

13 Aruoma OI, Halliwell B, Hoey BM and Butler J: The antioxidant action of $\mathrm{N}$-acetylcysteine: its reaction with hydrogen peroxide, hydroxyl radical, superoxide, and hypochlorous acid. Free Rad Biol Med 6, 593-597 (1989)

14 Wasynczuk A, Kirksey A and Morré DM: Effects of maternal vitamin B-6 deficiency of specific regions of developing rat brain: the extrapyramidal motor system. J Nutr 113, 746-754 (1983)

15 Guilarte TR and Wagner HN Jr: Increased concentrations of 3hydroxykynurenine in vitamin $\mathrm{B} 6$ deficient neonatal rat brain. J Neurochem 49, 1918-1926 (1987) 